\title{
La Sculpture au défi. Surréalisme et matérialisme
}

\author{
Sculpture chalenge. \\ Surrealism and materialism
}

\author{
DIDIER OTTINGER \\ Centre national d'art et de culture Georges-Pompidou (France)
}

Recibido : 2-V-2015 Aceptado : 26-V-2015

\section{RÉSUMÉ}

En prenant comme point de départ la proposition d'André Breton d'une «physique de la poésie ", nous analyserons ici les conditions de surgissement de diverses variations qui pris forme dans la pratique surréaliste de l'objet surréaliste, depuis la Boule suspendue (1930) de Giacometti aux sculptures crées par Miró dans les années cinquante, en prenant compte de la construction des objets à fonctionnements symboliques de Dalí, à l'« Équation de l'objet trouvé», écrit fondamental de Breton, et à la célébration, en 1936, de l'exposition fondationale à la Galerie Charles Ratton.

\section{MOTS CLÉS}

OBJET SURRÉALISTE, BRETON, SCULPTURE, COLLAGE

\begin{abstract}
Taking as the starting point the Bretonian proposed «physique de la poésie», we analyse the conditions of emergence of the different variations that meet the Surrelist practice of Surrelism object, from the Boule suspendue (1930) of Giacometti, until the sculptures created by Miró in the fifties, focused on the construction of objets à fonctionnements symboliques by Dalí, the fundamental writing of Breton about the «Equation de l'objet trouvé» and the celebration, in 1936, of the founding exhibition at the Galery Charles Ratton.
\end{abstract}

(C) Contrastes. Revista Internacional de Filosofia, vol. XXI-N³ (2016), pp. 161-176. ISSN: 1136-4076

Departamento de Filosofía, Universidad de Málaga, Facultad de Filosofía y Letras Campus de Teatinos, E-29071 Málaga (España) 


\author{
KEYWORDS \\ SURREALIST OBJECT, BRETON, SCULPTURE, COLLAGE
}

De même que la physique contemporaine tend à
se constituer sur des schèmes non-euclidiens,
la création des « objets surréalistes » répond
à la nécessité de fonder, selon l'expression
décisive de Paul Éluard, une « véritable
physique de la poésie ».
André Breton, « Crise de l'objet»

I

UN SECOND CHAPITRE DE L'HISTOIRE DU SURRÉALISME s'ouvre au début de l'année 1927 avec l'engagement de ses membres les plus actifs (André Breton, Louis Aragon, Paul Éluard, Pierre Unik, Benjamin Péret) dans les rangs du Parti Communiste Français. Lors de sa fondation, trois années plus tôt, le Surréalisme s'était donné un intitulé qui affirmait sans ambages son programme d'un dépassement, d'une réinvention du réel. La ligne poétique définie par Breton dans son Manifeste fondateur, en appelait au « modèle intérieur », aux puissances du rêve et de l'inconscient, que symbolisaient les yeux clos du personnage du Cerveau de l'enfant (1914) de Giorgio de Chirico. Dans les pages des premiers numéros de la Révolution Surréaliste, Antonin Artaud multiplie les invectives contre la « matière », contre ceux qui lui sacrifiaient toutes « puissances d'évasion »; André Breton invite à « Lâcher systématiquement la proie pour l'ombre ». L'automatisme, graphique et scripturaire s'imposait comme l'accélérateur le plus efficace pour déborder, pour affoler le réel, pour en ébranler toutes représentations rationnelles.

Le réel renâclait à disparaitre à la seule injonction de paupières qui se closent. Le rapprochement des surréalistes avec le parti communiste devait bientôt chausser leur idéalisme de semelles de plomb. Ses lectures de Lénine et de Trotski conduisent Pierre Naville à entrer en contact avec les jeunes communistes de la revue Clarté. Directeur des premiers numéros de la Révolution Surréaliste, il devient, en 1926, celui de Clarté. La révolution et les intellectuels (que peuvent faire les surréalistes), qu'il rédige la même année, prône un rapprochement entre surréalisme et marxisme. C'est leur opposition à la guerre du Rif (au Maroc) qui rapproche les surréalistes des membres de la revue Clarté. Le Lénine de Léon Trotski que découvre André Breton agit comme un révélateur. (Note : l'ouvrage est publié en 1925 par la Librairie du Travail). « Il n'est pas niable que, si la lecture d'un tel ouvrage m'avait transporté, c'est surtout 
par son côté sensible que j'avais été pris. D'un certain rapport de l'humain, la personne même de Lénine telle que l'auteur l'avait intimement connue, au surhumain (la tâche qu'il avait accomplie) se dégageait quelque chose de très entraînant qui, du même coup, conférerait aux idées qui avaient été les siennes le plus grand pouvoir d'attraction » (Breton 1999, t III, 502-503). Le poète livre un compte-rendu enthousiaste de sa lecture dans le ${ }^{\circ} 5$ d'octobre 1925 de $L a$ Révolution surréaliste. (" Ce livre est un des plus grands que j'ai jamais lus ». confirme Paul Éluard dans une lettre adressée à Breton en septembre 1925).

Le ralliement des surréalistes au PC leur impose de composer avec un réel qui constitue le socle théorique, philosophique, de l'idéologie communiste. Conscient du virage que se doit de prendre son mouvement, André Breton en appelle à la fondation d'une «physique de la poésie ».

C'est dans les pages de Hegel qu' André Breton entrevoie une synthèse possible entre poésie et politique : «Breton avait d'emblée esquissé pour l'essentiel, et en travaillant sur la matière poétique elle-même, l'Aufhebung, l'absorption et le dépassement de l'art et de la littérature dans une conception plus large de la création artistique, et entrevu la possibilité d'une pratique poétique axée sur le bouleversement de la vie quotidienne » note Louis Janover $(1980,16)$. Si la nouvelle revue du surréalisme, Le Surréalisme au service de la révolution, affirme une allégeance sans réserve, sa couverture s'orne d'un signe astrologique et alchimique qui ne peut que conforter le soupçon d'idéalisme que les autorités $\mathrm{du}$ PC font peser sur Breton et ses amis. L'évolution de Pierre Naville n'est pas de nature à rassurer le $\mathrm{PC}$ quant à l'orthodoxie communiste des surréalistes. En 1927, Naville se rend en Russie où il peut rencontrer Léon Trotski. A son retour, il ne tarde pas à infléchir la ligne idéologique de la revue Clarté pour en faire l'organe d'une opposition de gauche au PCF. Cette évolution lui vaut d'être exclu du PC en 1928.

Mis en demeure au Congrès international des écrivains révolutionnaires de Kharkov de novembre 1930, Louis Aragon et Georges Sadoul acceptent d'abjurer freudisme et « trotskisme » dont ils se voient accusés. En gage de son orthodoxie, André Breton publie Les Vases communicants (1932), un ouvrage qu'André Thirion interprétera des années plus tard comme « la préface d'une action militante $»,{ }^{1}$ comme le témoignage d'une adhésion au matérialisme dialectique.

Les jeunes poètes du Grand Jeu deviennent les victimes expiatoires de ce contexte de suspicion dans lequel le surréalisme est amené à justifier idéologi-

1 «Breton achevait Les Vases communicants qu'il méditait d'écrire depuis le retour de Kharkov et qu'il considérait comme la préface d'une action militante [...] Breton a écrit cet essai pour témoigner qu'il s'est défait de toute attache idéaliste. » (Thirion 1999, 518). 
quement. Dénonçant les dérives de René Daumal et Roger Gilbert-Lecomte vers la « théosophie, les paradis artificiels, la croyance aux mondes suprasensibles et aux pratiques divinatoires » (Thirion 1999, 338), Thirion pense dédouaner le surréalisme de ces mêmes reproches qui lui sont adressés: « ...l'arrivée chez les surréalistes de quelques petits apprentis sorciers du genre Daumal risquait de renforcer la tendance aux mystères et de compromettre définitivement une adhésion véritable du groupe au matérialisme historique », relatera Thirion $(1999,310)$, non sans un certain cynisme.

Décidant de se placer sans réserves " au service de la Révolution », le surréalisme reconsidère son iconoclasme hérité de dada à l'aune de son engagement politique. La contestation de la fétichisation de l'œuvre d'art, la remise en cause dadaïste de la mythologie du génie, se voit investie d'un sens, éthique, révolutionnaire.

Dans L'Idéologie allemande [1845-46, publié en 1932], Karl Marx et Friedrich Engels avaient écrit que : « La concentration exclusive du talent artistique dans quelques individus et, corrélativement, son étouffement dans la grande masse, est une conséquence de la division du travail »; ils poursuivaient « Dans une société communiste, il n'y a plus de peintres, mais tout au plus des hommes qui, entre autres, font de la peinture » (cité par Janover 1980, 64). André Breton avait pu trouver une première équivalence poétique de ces analyses politiques dans les écrits de Lautréamont prônant un art « fait par tous ». Le jeu du Cadavre exquis institué comme pratique privilégiée à partir de 1925, l'écriture à plusieurs mains (initiée par Les champs magnétiques de 1919), apportaient une première, bien qu'insatisfaisante réponse aux conjectures de Marx. Trop de subjectivité, trop de soupçon de « talent » subsistaient encore dans les productions plastiques et littéraires du Surréalisme. Si l'existence d'une caste artistique entérinait l'aliénation d'un prolétariat amputé de ses facultés créatrices, le commerce des œuvres exemplifiait, lui, une fétichisation de la marchandise, une spéculation financière qu'avait dénoncées Marx et Engels dans le Manifeste du Parti Communiste (1848) : « [...] toutes les activités dites supérieures, spirituelles, artistiques, etc., étant devenues des articles de commerce, elles ont perdu leur ancien prestige » (cité par Janover 1980, 57). Faisant écho à cette analyse, Pierre Naville était allé jusqu'à affirmer dans la troisième livraison de la révolution Surréaliste que : « Plus personne n'ignore qu'il n'y a pas de peinture surréaliste. Ni les traits du crayon livré au hasard des gestes, ni l'image retraçant les figures de rêve, ni les fantaisies imaginatives, c'est bien entendu, ne peuvent être ainsi qualifiées » (Naville 1926, 85). Un peu plus tard, amené à théoriser la relation entre surréalistes et communisme, il précisait : « la vie du rêve, celle de l'amour, les différentes occupations artistiques, littéraires, pseudo-philosophique auxquelles les surréalistes se sont livrés, et qu'ils considèrent de leur propre aveu comme primordiales, sous leur 
apparence révolutionnaires et libérés des liens bourgeois, gardent toujours certains refuges, certains retraits mystiques, des attaches beaucoup trop facilement acceptés avec ce monde bourgeois » (Naville 1926, 85). Les réserves formulées par Naville à l'endroit d'une production artistique surréaliste qu'il juge encore tributaire des critères de l'art « bourgeois » ne sont pas entendus, compris, par un surréalisme encore politiquement «immature ». Au déni péremptoire de Naville quant à la possibilité même d'une peinture surréaliste André Breton répondra par un long essai Le surréalisme et la peinture qu'il livrera en « feuilleton » à La Révolution surréaliste. Quelques années plus tard, l'engagement politique viendra ébranler ce bel édifice esthétique...

Au début des années trente, comme Lénine en 1902, les surréalistes se voient confronter à une seule question : Que faire? Quelle forme donner à leurs œuvres qui se doivent désormais de contester talent et savoir-faire, qui doivent viser à combattre toutes formes de génialité individuelle, résister à la fétichisation marchande?

Une première réponse est apportée par Aragon, qui organise en mars 1930, à la galerie parisienne de Camille Goemans, une exposition qu'il intitule $L a$ Peinture au défi. Le « défi » est celui que constitue la pratique du collage qui, écrit Aragon, «met en question la personnalité, le talent, la propriété artistique, et toutes sortes d'autres idées qui chauffaient sans méfiance leur pieds tranquilles dans les cervelles crétinisées » (Aragon 1965, 42). Et l'auteur du Paysan de Paris d'alléguer l'exemplarité de Francis Picabia et de Marcel Duchamp qui, à coup de ready-mades ou de collages de spaghettis, ont saboté le prestige de la peinture, instruisant par leur iconoclasme « le procès de la personnalité ». Lautréamont est invoqué par Aragon, pour avoir prophétisé l'avènement d'un art, d'une poésie « faite par tous. Non par un » (Aragon 1965, 71). Faisant écho aux analyses de Marx sur la dégradation, sur la fétichisation des œuvres de l'esprit opérées par le capitalisme, Aragon dénonce une peinture «luxueuse », un tableau devenu « bijou », avant de conclure : « voici qu'il est possible aux peintres de s'affranchir de cette domestication par l'argent. Le collage est pauvre » (Aragon 1965, 57).

En dépit de ses vertus « politiques », le collage ne parvient pas à s'imposer comme le sésame ouvrant aux surréalistes les portes du matérialisme dialectique. C'est au duo Dalí-Thirion (à l'artiste qui, aux yeux de Breton, « incarne l'esprit surréaliste » et au garant de l'orthodoxie communiste) qu'est confiée la mission de résoudre le problème qui reste en suspens. «Dalí et moi essayions de trouver des points d'ancrage à partir desquels chaque surréaliste pourrait exercer son talent vers une direction commune, dans le cadre d'une discipline acceptée par tous. Une de mes préoccupations était d'éviter un dérapage de l'intérêt que nous portions à la psychanalyse, aux rapports du conscient et de l'inconscient, vers des affirmations philosophiques que nos adversaires qualifieraient d'idéa- 
listes et donneraient de la consistance aux accusations de freudisme [...] Dalí proposa d'entreprendre la fabrication d'objets à fonctionnement symbolique » (Thirion 1999, 512-513). L'objet était bien la solution attendue. Mieux que le collage, qui restait une interprétation du réel, en laquelle subsistait une part d'expression subjective, l'objet constituait le réel lui même, le fondait, sans interprétation ni médiation.

Les objets soit ; mais lesquels? Au moment même où Thirion et Dalí engagent leur réflexion visant à répondre au rappel à l'ordre matérialistes du PC, Alberto Giacometti réalise sa Boule suspendue (1930). L'érotisme manifeste, la forme inédite de la « Boule »-qui tient davantage du jeu pour enfant que de la statuaire classique- la destinent, pour un temps, à incarner la réponse espérée par Breton et les siens. Prototype des «Objets à fonctionnement symbolique », avec la Boule suspendue s'ouvre l'histoire de l'objet surréaliste.

\section{LA BOULE SUSPENDUE}

Les femmes-cuillers que présente Alberto Giacometti à la galerie Jeanne Bucher en 1928 sont remarquées par André Masson qui approche le sculpteur, le met en contact avec les familiers de son atelier de la rue Blomet: Antonin Artaud, Georges Bataille, Michel Leiris, André Queneau. L'année suivante, Giacometti rejoint le cercle qui se forme autour de la revue Documents crée par l'historien de l'art primitif Carl Einstein (en 1915, Einstein avait rédigé un ouvrage pionnier consacré à la sculpture africaine, Negerplastik) et Georges Bataille. Membre du comité de rédaction de Documents, Michel Leiris lui consacre en 1929 son premier texte critique. Homme et femme (1928-1929), Cage (1930-1931), que réalise alors Giacometti s'emparent des thématiques tragiques, sacrificielles, qui caractérisent l'orientation que Bataille donne à sa revue. Quand ses sculptures récentes sont présentées au printemps 1930 à la galerie Pierre Loeb, Salvador Dalí attire sur elles l'attention d'André Breton qui, sans délai, propose au sculpteur de rejoindre les rangs du Surréalisme. Parmi ces œuvres figure la Boule suspendue.

Dalí en éclaire le sens, en décembre 1931, dans le troisième numéro de la revue Le Surréalisme au Service de la Révolution, avant d'en faire le prototype des objets à fonctionnement symbolique. La Boule suspendue tient davantage de l'objet, plus précisément du jouet, que de ce que le sens commun avait jusque-là défini comme sculpture. À l'instar des œuvres conçues durant les mêmes années par Giacometti-Main prise (1932), Circuit (1931), Pointe à l'œil (1931-1932)-, la Boule suspendue est animée, ou peut potentiellement l'être, d'un mouvement réel. Un mécanisme simplifié invite à sa manipulation, suscite son appréhension tactile. Ne serait-ce que par cette seule adresse haptisch, la Boule suspendue procéde à un déclassement, à une démystification de l'œuvre 
d'art (qu'on « Ne touche pas ! »), qui s'accorde au projet de « défétichisation » auquel entend s'atteler le surréalisme.

Objet -à fonctionnement-symbolique, l'œuvre est riche d'une charge d'inconscient, relève d'une évidente signification érotique. Si, comme le suggère Yves Bonnefoy, Giacometti a pu emprunter aux Danseuses espagnoles (1928) de Miró leur légèreté ludique, voire certains des objets qui les composent (boule, fils...), ${ }^{2}$ la Boule suspendue doit davantage au « sadisme » de Bataille qu'à l'humour subtil de Miró. Parmi les premiers, Maurice Nadeau rendra compte (en 1945) du malaise que lui inspirent la Boule suspendue et son mouvement contrarié, suggérant la répétition sans fin d'un « échec ». Conçue alors que Giacometti est familier du cercle de Bataille, la Boule suspendue est rapprochée par l'historienne américaine Rosalind Krauss du sadisme, de la violence sacrificielle qui constitue la « ligne théorique » de la revue Documents (Krauss 1985, v. 2, 511-512). L'œuvre fait pour elle écho à l'œil fendu par une lame de rasoir du film Le Chien andalou (1929) de Luis Buñuel, renvoie à la scène de l'œil énucléé, glissé dans un sexe féminin, de l'Histoire de l'œil (1928) de Georges Bataille.

L'érotisme inhérent à la Boule suspendue, sa forme, jugée « extraplastique » par Dalí, sa mobilité potentielle, établissent la définition des objets à fonctionnement symbolique.

\section{OBJETS À FONCTIONNEMENT SYMBOLIQUE}

La banalisation du geste artistique, que les surréalistes s'efforcent d'imposer à leur production, conduit Dalí et Thirion à surévaluer la dimension « extra-plastique » de la Boule suspendue. Seule une remise en cause radicale du savoir-faire de Giacometti pouvait garantir l'exemplarité « démocratique » des objets à fonctionnement symbolique. « La fabrication de ces objets offrait à tous les non-artistes la possibilité d'exprimer plastiquement leurs rêves, leurs fantasmes, leurs désirs, bref, leur capacité d'invention et de création mutilée par une éducation spécialisée et par l'idiotisme des métiers », constate Janover $(1980,47)$. Thirion croit à cette universalité du talent artistique, au point d'envisager d'assembler son propre objet : « je pensais à l'objet à signification symbolique que j'avais imaginé et que je n'avais pas construit, faute d'argent [...] En voici l'argument : une boule, suspendue en équilibre en haut d'un plan incliné (une planchette). Sur une faible impulsion la boule aurait pu descendre le plan incliné et se loger en partie dans une cavité de même forme, tapissée de mèches de coton» (Thirion 1999, 525-526).

2 «C'était la grande liberté. Quelque chose de plus aérien, de plus dégagé, de plus léger que tout ce que j'avais vu. En un sens c'était absolument parfait. », (Giacometti cité par Bonnefoy 1990, 193). 
S'il revient à Thirion d'affirmer le caractère anartistique des objets à fonctionnement symbolique, c'est à Dalí d'en souligner la nature surréaliste, d'en préciser les liens avec les forces de l'inconscient :

Ces objets, qui se prêtent à un minimum de fonctionnement mécanique, sont basés sur les phantasmes et représentations susceptibles d'être provoqués par la réalisation d'actes inconscients [...] Les objets à fonctionnement symbolique ne laissent nulle chance aux préoccupations formelles. Ils ne dépendent que de l'imagination amoureuse de chacun et sont extra-plastiques [...] La culture de l'esprit s'identifiera à la culture du désir (Dalí 2012, 348).

Libérant sa verve de bonimenteur, Dalí se fait l'apôtre de la diffusion de tels objets :

Je me mis alors en campagne pour lancer l'objet surréaliste, l'objet irrationnel à fonctionnement symbolique, contre les récits de rêve et l'écriture automatique, etc. L'objet surréaliste devait être absolument inutile tant au point de vue pratique que rationnel. Il matérialisait, avec le maximum de tangibilité, les fantaisies spirituelles de caractère délirant. La présence et la circulation de ces objets concurrencèrent l'objet utile et pratique, avec une telle violence que l'on crut assister à une bataille de coqs enragés dont l'objet normal sortait le plus souvent déplumé. Les appartements parisiens vulnérables au Surréalisme s'encombrèrent rapidement d'objets surréalistes, déconcertants à première vue, mais que chacun pouvait toucher et manipuler de ses propres mains. On venait palper le corps nu et sortant de mon puits, de cette vérité catholique que l'objet est un «état de grâce» (Dalí 2012, 348).

\section{CONTENU MANifeste ET CONTENU LATENT}

Au milieu des années trente, l'objet à fonctionnement symbolique disparait du paysage surréaliste avec celui qui en aura été le plus ardent promoteur. En 1933, Dalí peint L'Enigme de Guillaume Tell, qui présente un Lénine agenouillé dont l'anatomie s'épanche en formes « molles». L'œuvre assimile le révolutionnaire bolchévique à la figure du père castrateur, jetant un éclairage ironique et lucide sur la relation chaque jour plus complexe entre le surréalisme et le parti communiste. Avec son poème « Front rouge », Aragon tranche le nœud gordien de cette relation en acceptant de mettre sa poésie au service de la propagande, et en acceptant la rupture avec le surréalisme corrélative à cette soumission. La lucidité ironique de Dali conduit elle aussi, en 1934, à son exclusion du surréalisme. L'objet à fonctionnement symbolique disparait avec son inventeur... 
André Breton ne tarde pas à dénoncer dans ces assemblages leur trop plein d'inconscient explicite, de désir trop explicitement affiché, le surcodage de leur « contenu manifeste », qui en fait autant de monument de ce subjectivisme, de cet « individualisme » par lesquels se distinguent les productions d'une culture « bourgeoise ».

Une visite du marché aux Puces de Saint-Ouen, qu'il effectue en compagnie de Giacometti en 1934, lui offre l'occasion d'une nouvelle charge contre l'artisanat, le savoir-faire, l'intentionnalité artistique résilientes des objets à fonctionnement symbolique. L' « objet trouvé » apparait à Breton comme capable de résoudre l' « équation » dont surréalisme et communisme constituent les deux inconnus. Il remet en cause les premiers objets surréalistes, qui « livrent à l'interprétation une étendue moins vaste [...] que les objets dans le même sens moins systématiquement déterminés. L'incorporation volontaire du contenu latent - arrêté d'avance - au contenu manifeste est ici pour affaiblir la tendance à la dramatisation et à la magnification dont se sert souverainement, au cas contraire, la censure » (Breton 2005,109).

Pour entériner ce dépassement des objets trop «systématiquement déterminés », Breton parvient à faire de leur créateur, Giacometti, l'inventeur d'un ready-made ! Il importait symboliquement à Breton que celui là même qui, avec sa Boule suspendue, avait été à l'origine des objets à fonctionnement symbolique consente à sacrifier son génie, son savoir-faire, sur l'autel du matérialisme dialectique et de ses implications esthétiques.

Giacometti rapporte des Puces de Saint-Ouen une visière de protection utilisée pendant la Première Guerre mondiale ; Breton y fait l'acquisition d'une cuiller en bois artisanale, qu'il décrira comme le substitut de la « pantoufle de vair », dont il avait commandé sans succès la réalisation au sculpteur. Libérés des « préoccupations formelles » encore attachées aux objets à fonctionnement symbolique, les objets trouvés aux Puces déploient les ailes d'une poésie pure. André Breton les identifie aux « objets de rêve » échoués dans le monde réel, dont il annonçait l'apparition en 1932 ( dans Les Vases communicants), en fait les messagers du « hasard objectif», ces révélateurs des destinées individuelles.

Il est possible que Giacometti ai tiré de sa pérégrination à saint Ouen des conclusions toutes autres... resté proche d'Aragon et de Crevel après leur soumission aux directives du Parti et leur rupture avec Breton, il confie en 1935 à la revue Commune, proche d'Aragon, des dessins dont le caractère propagandistes continus d'interroger les exégètes du sculpteur. La même année, dans son message « aux peintres », Crevel dénonçait : " l'escroquerie du scandale formel, la rage d'originalité à tout prix » (Bonnefoy 1990, 247). Il est troublant de mettre en regard la synchronie du retour de Giacometti au modèle vivant (au réel ?) et la rupture définitive de Breton avec un PCF qui entendait imposer sa conception réaliste de l'art. Loin de tout déterminisme lié au mouvement 
interne de son art, à un possible retour au giron familial ou académique, Giacometti aurait-il choisi un camp plus politique que purement biographique?

\section{L'EXPOSITION SURRÉALISTE D'OBJETS À LA GALERIE CHARLES RATTON}

L'Exposition surréaliste d'objets, inaugurée le 22 mai 1936 à Paris, galerie Ch. Ratton, n'a (presque) plus rien d'une « exposition d'objets surréalistes ». Les épreuves manuscrites du catalogue témoignent de l'hésitation d'André Breton quant à l'intitulé de l'exposition. D'un titre l'autre se dit l'instabilité, le flottement du «Surréalisme », appliqué aux objets concrets en ce milieu des années 1930. Les objets exposés chez Ratton sont-ils « surréalistes » par nature, ou le deviennent-ils sous l'effet du regard « surréaliste » porté sur eux ? À quel titre les objets « mathématiques », « naturels », « trouvés et interprétés », ceux d'Amérique ou d'Océanie, un grand tamanoir, une bouteille trouvée après l'éruption du Mont Pelé, une fougère... sont-ils « surréalistes »?

Pour devenir « surréaliste », démontre Breton en 1936, un objet n’a à subir aucune métamorphose, aucune altération. La transsubstantiation surréaliste de l'objet s'éclaire de la définition du ready-made, que livre Breton, dans le Dictionnaire abrégé du Surréalisme (1938). Ce « premier objet surréaliste » est « objet usuel promu à la dignité d'objet d'art par le simple choix de l'artiste ». Oublions ce que cette définition peut avoir de partielle, (feignant d'ignorer qu'un ready-made est toujours constitué d'un objet et d'un texte) pour n'en considérer que la dimension « magique » (« qui sort du rationnel » précise le dictionnaire Larousse). Le « choix », l'élection de l'artiste (à condition toutefois qu'il soit surréaliste), suffit à métamorphoser un objet quelconque en un « objet surréaliste » (soit presque un objet « d'art»). Ayant tous, sans exception, été choisis par des surréalistes, les objets exposés en 1936 à la galerie Ratton sont, de fait, des « objets surréalistes ». L'exposition qui les regroupe a, dès lors, toutes les apparences d'une « exposition d'objets surréalistes ».

En dépit de cette logique apparemment implacable, Breton a tenu à ce que l'exposition de la galerie Ratton soit une « exposition surréaliste d'objets ».

« L'Exposition surréaliste d'objets » est vouée à la quintessence d'un Surréalisme qui démontre avec elle, sa capacité à transmuter la nature des objets les plus improbables (l'ombre de l'alchimie plane sur cette thaumaturgie), à transfigurer le réel. La puissance de désignation surréaliste, qui constitue l'objet même de l'exposition, aurait pu aussi bien conduire à la titrer : « Exposition du Surréalisme ».

L'exposition « Ratton » constitue le point d'orgue de la réflexion surréaliste appliquée à l'objet. Elle se place à l'apogée d'une trajectoire retraçant un processus de conceptualisation, celui de l'affirmation d'un Surréalisme rendu à sa pureté poético-théorique. 
En renonçant à être une « exposition d'objets surréalistes », l'exposition résout les apories auxquels se heurte le surréalisme dans sa relation à la politique. Dans les vitrines, sur les murs de la galerie Ratton, nulles traces (ou presque), du savoir faire, du talent, du génie caractéristiques de l'âge esthétique bourgeois. Ready made sorti momentanément de leur anonymat fonctionnel ces objets défient la spéculation marchande (à l'instar du « ceci n'est pas un morceau de fromage de Renée Magritte démembre à l'issue de l'exposition, dont la cloche à fromage est rendue à son usage premier).

Suprême sortilège de la « dialectique », c'est par la célébration du réel dans ce qu'il a de plus « obtus », en s'attachant aux objets les plus ordinaires que s'accomplit l'efflorescence d'un Surréalisme rendu à sa forme la plus abstraite, la plus chimiquement pure.

L'« idéalisation » de l'objet surréaliste que consacre l'exposition Ratton est symptomatiquement contemporaine du virage politique qui, après la rupture avec le $\mathrm{PC}$, devait conduit Breton à se rapprocher de Trotski. Au réalisme socialiste stalinien, se substituera bientôt la « totale liberté de création de l'artiste » affirmée dans le Manifeste pour un art révolutionnaire indépendant » que cosigneront Trotski et Breton en 1938. En phase avec cette « liberté » réaffirmée, le surréalisme de la fin des années trente renoue avec l'automatisme du premier Manifeste auquel les inventions techniques de Paalen (fumages), de Dominguez (décalcomanie), redonne une actualité.

Dans le «communiqué de presse » qu'il prend soin de rédiger pour l'exposition de la galerie Ratton, Breton établit la taxinomie des objets exposés :

« objets naturels », minéraux (cristaux contenant de l'eau plusieurs fois millénaire), végétaux (plantes carnivores), animaux (tamanoir, œuf d'œpyornix), des « objets naturels interprétés » (un singe en fougère) ou « incorporés » à des sculptures, des « objets perturbés » (c'est-à-dire modifiés par des agents naturels, incendie, tempêtes, etc.) [...] plusieurs objets venus de l'atelier de Picasso, qui prennent place, historiquement, avec les célèbres « ready-mades » et « ready-mades aidés » de Marcel Duchamp, également exposés. Enfin les objets dits « sauvages », les plus beaux fétiches et masques américains et océaniens [...] Les « objets mathématiques », surprenantes concrétisations des plus délicats problèmes de géométrie dans l'espace, et les « objets trouvés » et « objets trouvés interprétés », nous conduisent aux « objets surréalistes » proprement dits (Breton, t. II, 1199-1200).

Cette liste annonce celle des objets qui composeront le « mur» qu'André Breton dans son atelier de la rue Fontaine après la Seconde Guerre mondiale. Comme le fera le « mur », l'exposition de la galerie Ratton « surréalise » les anciennes chambres de merveilles, Wunderkammern et autres cabinets de curiosité. Monuments d'un savoir préscientifique, ils évoquent un temps durant lequel intuition poétique et connaissance rationnelle cohabitaient encore (une 
compatibilité qu'André Breton verra à nouveau éclore dans les théories de la physique quantique). Les chambres de merveilles regroupaient les objets les plus hétéroclites selon les rubriques de l'artificialia : objets créés ou modifiés par l'homme, antiquités, œuvres d'art; des naturalia : créatures et objets naturels (et tout spécialement les monstres); des exotica : plantes et animaux exotiques ; des scientifica : outillages et appareils scientifiques ; toutes catégories recoupant celles des objets exposés chez Ratton.

Parmis ceux alignés dans les vitrines de la galerie, les « objets mathématiques » (ces modernes scientifica), découverts par Max Ernst à l'Institut Poincaré, possèdent un statut exemplaire. Donnant une forme visible à des équations mathématiques complexes, ils illustrent au plus haut point la vocation des objets surréalistes à fonder une « physique de la poésie », à ancrer les idées dans le réel. Ils rendent explicite la nature des objets trouvés surréalistes : concrétions de rêves et de désirs.

Dans le numéro des Cahiers d'art consacré à l'objet, publié au moment de l'exposition, Breton publie « Crise de l'objet», dans lequel il établit un lien direct entre l'exposition et les théories scientifiques contemporaines qui renouent avec le principe de continuité des savoirs anciens : « la raison d'aujourd'hui ne se propose rien tant que l'assimilation continue de l'irrationnel...» (Breton t. IV 2008, 21). De l'annonce d'une prochaine « exposition d'objets surréalistes » publié dans livraison du $1^{\mathrm{er}}$ décembre 1926 de la Révolution surréaliste, à l'« Exposition surréaliste d'objets » se mesure le chemin, poétique autant que politique, accompli en une décennie par le Surréalisme.

\section{L'OBJET AU DÉFI DE LA SCULPTURE}

La Seconde Guerre mondiale conduit nombre de surréalistes à l'exil. André Breton, Max Ernst, André Masson, Roberto Matta, Yves Tanguy... s'installent aux États-Unis. Alors que dans une France occupée la revue surréaliste, $\mathrm{La}$ Main à plume, fidèle aux réflexions initiées durant les années trente, entreprend (en 1943) de consacrer un numéro spécial à l'objet (qui ne paraîtra pas), à Long Island, Max Ernst travaille à l'intégration de l'objet à une sculpture qui retrouve une forme conventionnelle (un anthropomorphisme qui la rattache même lointainement à la statuaire classique).

Les années 1940 voient l'apparition d'une nouvelle génération de sculptures pour lesquelles l'objet ordinaire, quotidien, devient le matériau élémentaire d'assemblages dont la logique constructive est celle du Cadavre exquis. Les moulages de plâtre de ses objets domestiques (bols, assiettes...), constituent le matériau premier des figures que façonne Max Ernst. Picasso, en 1942, assemble une selle et un guidon de vélo, pour former un « Taureau » (dont la photographie devait orner la couverture du numéro de la Main à plume consacré à l'objet...). 
En contact avec Joan Miró dès 1932, Alexander Calder avait élargie son vocabulaire formel à un biomorphisme inspiré du végétal et de l'animal (ses gouaches évoquant des organismes en croissance ou en mutation, réalisées cette même année, témoignaient de cette inspiration nouvelle). Rompant avec son modèle technique de prédilection, un ensemble de sculptures de 1935-1936, assemblant des pièces de bois polies, évoquait les œuvres de Arp, les objets de Miró. 1936 marquait le point d'orgue du rapprochement de Calder et du Surréalisme. Ces œuvres figuraient à New York, dans l'exposition d'Alfred Barr Fantastic Art, Dada, Surrealism, à Londres, dans l'« Exposition internationale du Surréalisme ». A Paris, un de ses mobiles était présenté dans l'« Exposition surréaliste d'objets » de la galerie Ratton. Deux ans plus tard, Apple Monster, assemblage des branches de pommier, fait référence au naturalisme, à la tératologie surréaliste.

Pablo Picasso est un des protagonistes majeurs de cette sculpture d'assemblage avec laquelle s’identifie la sculpture surréaliste. Dès 1912, il avait introduit dans ses œuvres des objets puisés dans son environnement quotidien (Nature morte à la chaise cannée) Son Verre d'absinthe, de 1914, incorpore une cuiller réelle. Ce recours aux objets quotidiens devient significatif de l'œuvre qu'il développe au début des années 1930, alors qu'il se rapproche du Surréalisme. La Tête de femme, de 1929-1930, se développe autour d'un égouttoir à salade. Ses reliefs sablés de 1930 se composent d'objets hétéroclites : gants, brindilles, morceaux de bois... Profil, un an plus tard, greffe une racine et un plumeau à une plante en pot. La Vénus du gaz (1945) n'est plus qu'un brûleur de cuisinière placé en position verticale. Combinant ready-made et collage, Picasso réalise durant les années 1950, des sculptures qui agrègent des objets quotidiens (un panier et de vraies chaussures pour la Petite Fille qui saute à la corde (1950), des voitures miniatures pour La Guenon et son petit (1951).

Brassaï a photographié les vitrines de l'atelier de Picasso dans lesquelles le peintre regroupait ses sculptures de petits formats. Comme dans les vitrines de la galerie Ratton, des moulages de Vénus aurignaciennes voisinent avec ses propres figures féminines, le verre d'absinthe avec les bouteilles et verres déformés par la chaleur de l'éruption de la Montagne Pelée...

L'exposition Le Surréalisme en 1947, organisée à la galerie Maeght, signe le retour de l'activité surréaliste en Europe. Elle reste fidèle au principe d'un dépassement de l'art qui avait justifié, avant-guerre, l'invention de l'objet surréaliste. Dans la préface du catalogue, Breton mentionne les « œuvres poétiques et plastiques récentes », qui « disposent sur les esprits d'un pouvoir qui excède en tous sens celui de l'œuvre d'art». Ce « pouvoir » est alors identifié à la capacité de ces « œuvres récentes » à constituer le ferment d'une mythologie nouvelle. Au cœur de l'exposition est aménagée une salle regroupant des « au- 
tels » consacrés à « un être, une catégorie d'êtres ou un objet susceptible d'être doué de vie mythique ».

L'ésotérisme prêté à ces objets est le nouvel argument qu'allègue le Surréalisme pour nier leur dimension esthétique. À l'instar des ready-mades de Duchamp, leurs ancêtres exotériques, ils sont avant tout des "psycho-objets », la cristallisation d'utopies collectives. Les conceptions esthétiques d'André Breton s'avèrent une fois encore en phase avec ses réflexions politiques. $\mathrm{Au}$ léninisme, au trotskisme des années trente succède, à l'issue de la seconde guerre mondiale, un intérêt d'André Breton pour le socialisme utopique de Charles Fourrier, précurseur de la pensée libertaire. Alerté par Pierre Naville, en juillet 1946, Breton est informé de l'existence d'un manuscrit du Nouveau Monde amoureux conservé à la bibliothèque de l'école normale supérieure. Sa lecture lui inspirera son Ode à Charles Fourrier publiée une année plus tard. Via Fourrier, Breton, et le surréalisme avec lui, renouent avec l'anarchisme de dada. En 1952, il confessera que « le surréalisme s'est pour la première fois reconnu [...] dans le miroir noir de l'anarchisme » (Naville 1975, 65). Dans Arcane 17, qu'il rédige en 1944, Breton réhabilite l'anarchisme de sa jeunesse : « Je n'oublierai jamais la détente, l'exaltation et la fierté que me causa, une des toutes premières fois qu'enfant on me mena dans un cimetière parmi tant de monuments funéraires déprimants ou ridicules la découverte d'une simple table de granit gravée en capitales rouges de la superbe devise Ni dieu ni maître ». De retour en Europe, le surréalisme opére un rapprochement effectif avec les fédérations anarchistes. Plusieurs des membres du groupe collaboreront à la revue Le Libertaire (12 octobre 1951-8 janvier 1953).

\section{VI. «RUPTURE INAUGURALE»}

La domination économique, qui est renforcée par la domination étatique et morale (comme la triade proudhonienne Capital, État et Église), la revendication de l'accomplissement des désirs et des passions, mais sans assujettissement aucun. Le rôle égalitaire, libre et actif, reconnu à la femme est un autre trait libertaire fouriériste. Assumer ses passions, les satisfaire pleinement, à son propre profit et à celui du collectif auquel on appartient, est un thème qui ne sera vraiment repris qu'au XXe siècle avec le freudisme et ses dérivés, surtout la gauche freudienne : notamment Wilhelm Reich (1897-1957), Erich Fromm (1900-1980) et plus tard Herbert Marcuse (1898-1979). Cet agencement libertaire, entre l'extrême diversité et la recherche d'une unité non réductrice et qui ne tend pas à l'uniformité. Un homme global, « universel », qui développe toutes ses dispositions et facultés, et qui assume toutes ses passions. Il n'y a donc pas opposition entre individu et collectif, mais interactions avantageuses pour les deux parties. C'est d'autant plus évident que la « concorde » est assurée en préservant le principe « d'affinité » que reprennent tous les anarchistes. 
Un post-fouriérisme, mettant en avant l'aspect libertaire, hédoniste, de liberté sexuelle... va être largement répandu dès le XIXe siècle.

Douze ans plus tard, en 1959, la VIIIe Exposition inteRnatiOnale $d u$ Surréalisme, à la Galerie Daniel Cordier, est tout entière dédiée à ÉROS, la puissance inspiratrice la plus secrète et la plus constante du mouvement. L'exposition embrasse une vaste chronologie, restituant à la Boule suspendue de Giacometti et à la Poupée de Bellmer une antériorité qui annonce le Bed de Robert Rauschenberg (le propre lit de l'artiste).

L'objet du couchant de Joan Miró trouve finalement sa place dans une exposition surréaliste. Miró l'avait conçu en 1935 (un dessin carnet FJM 13231411, Coll. Fondation Miro, reproduit p. 86 cat MNAM en précise la genèse) afin qu'il figure dans l'exposition surréaliste d'objets de la galerie Ratton. Son iconographie ouvertement sexuel, ses éléments mobiles : chaine, corde, ressort, en faisaient un parfait « Objets à fonctionnement symbolique ». Miró avait négligé de prendre en compte la condamnation tacite des Objets à Fonctionnement symboliques qui avait conduit à l'intitulé de l'exposition Ratton, passant d'une « exposition d'objets surréaliste » à une « exposition surréaliste d'objets ». En 1959, les « objets à fonctionnement symbolique » de Miró a cessé de poser un quelconque problème théorique. Dans la « Crypte du fétichisme », conçue par Mimi Parent pour l'exposition EROS, sont regroupés, dans des casiers muraux, une série d'objets qui vient rappeler l'érotisme consubstantiel à l'objet surréaliste.

Animé du farouche désir d' " assassiner la peinture », Joan Miró avait entrepris, en 1929, une série de Constructions que Jacques Dupin assimile à une entreprise de « mise en question d'un outil plastique trop facilement dominé, après les plongées dans les eaux troubles, les eaux, mères de l'inconscient et du rêve » (Dupin 1993, xx). Les sculptures que réalise Miró au milieu des années 1960 tenaient à la fois du collage et du ready-made. «Il ne récolte pas les choses comme un esthète pressé de jouer avec elles, de les bousculer, de les soumettre à son caprice, ni pour les intégrer à une vision de constructeur, non, il les transplante, telles quelles, il les accueille sur sa terre labourée, son aire de jeu... » (Dupin 1993, 372). Libérées des contraintes de l'idéologie, l'art surréaliste de l'objet surréaliste aimante à nouveau les parapluies et les machines à coudre, retrouve la grâce et l'humour du cadavre exquis. 


\section{RÉFÉRENCES BIBLIOGRAPHIQUES}

ARAGON, L. 1965 : « La peinture au défi », Les Collages. Paris : Hermann.

BONNEFOY, Y. 1990 : Giacometti. Paris : Flammarion.

BRETON, A. 2005 : «Équation de l'objet trouvé », «Intervention surréaliste », Documents

34, juin 1934 ; L'Objet surréaliste (textes réunis et présentés par Emmanuel Guigon). Paris : Jean-Michel Place.

Breton, A. 2008 : « Crise de l'objet », Cahiers d'art, n 1-2 [1936] ; O.C. Paris :

Gallimard, coll. « La Pléiade », t. IV.

BRETON, A. 2008 : préface au catalogue de l'« Exposition surréaliste d'objets », en O.C.

Paris : Gallimard, coll. « La Pléiade», t. II.

DALÍ, S. 1931 : «Objets surréalistes », Le Surréalisme au service de la révolution, $\mathrm{n}^{\circ}$

3, Paris, décembre 1931.

DALÍ, S. 2012 : La Vie secrète de Salvador Dalí. Paris : Gallimard.

DUPIN, J. 1993 : Miró. Paris : Flammarion.

JANOVER, L 1980 : Surréalisme, art et politique. Paris : Éditions Galilée.

KRAUSS, R. 1991: Le Primitivisme dans l'art du 20e siècle : les artistes modernes devant

l'art tribal. Paris: Flammarion, vol. 2.

NAVILLE, P. 1975 : La révolution et les intellectuels. Paris : Gallimard.

THIRION, A. 1999 : Révolutionnaires sans révolution. Paris : Actes Sud.

Didier Ottinger es Director adjunto del Centre Pompidou (Paris). 